УДК 623.418

\title{
ПРОЕКТИРОВАНИЕ И УПРАВЛЕНИЕ РАЗРАБОТКОЙ ЗЕНИТНЫХ РАКЕТНЫХ СИСТЕМ
}

Баштанник Николай Андреевич к.т.н., доцент Лобейко Владимир Иванович д.т.н., профессор Мустафаев Нияз Гаджикурбанович К.T.H.

Погребняк Иван Сергеевич соискатель ученой степени к.т.н. филиал Астраханского государственного университета в г. Знаменске Астраханской области

Аннотация: Зенитная ракетная система представляет собой сложную техническую систему. В данной работе рассматривается один из методов проектирования и основные принципы управления разработкой зенитных ракетных систем, а также некоторые этапы жизненного цикла живучести системы.

Ключевые слова: зенитная ракетная система, проектирование, разработка, управление, каскадная модель, спиральная модель, жизненный цикл.

\section{DESIGN AND DEVELOPMENT MANAGEMENT}

ANTI-AIRCRAFT MISSILE SYSTEMS

Bashtannik Nikolay Andreevich Lobeyko Vladimir Ivanovich Mustafayev Niyaz Hajikurbanovich Pogrebnyak Ivan Sergeevich 
Abstract: The anti-aircraft missile system is a complex technical system. This paper discusses one of the planning methods and the basic principles of managing the development of anti-aircraft missile systems, as well as some stages of the life cycle of the survivability of the system.

Key words: anti-aircraft missile system, planning, development, management, cascade model, spiral model, life cycle.

\section{Введение}

К сложным системам военной техники относят совокупность функционально связанных образцов или элементов образцов военной техники и средств обеспечения, объединенных для самостоятельного выполнения стратегической, оперативно-тактической и других задач. Зенитная ракетная система (ЗРС) представляет собой сложную систему [1].

Структура системы строится по иерархическому принципу: выполнение функций более высокого уровня обеспечивается совокупностью частных функций предшествующего уровня, которые, в свою очередь, обеспечиваются функциями более низкого уровня и так далее вплоть до элементарных функций, реализуемых отдельными элементами системы.

Основным свойством системы является ее целостность, достигаемая путем определенных взаимосвязей, взаимодействий подсистем (компонентов) и проявляющихся новых свойств, которыми компоненты системы не обладают.

Решение проблем проектирования зенитных ракетных систем требует применения комплексных методов исследования, позволяющих с единых позиций сравнивать различные варианты построения систем по совокупности их показателей качества и обеспечивающих выбор среди них наиболее предпочтительного.

В процессе проектирования систем используются математические, экспериментальные и эвристические методы. Выбор метода проектирования должен производиться на основе следующих основных направлений [2]:

— качества проектирования; 


\section{- стоимости проектирования;}

- сроков разработки;

— количества занятых специалистов-разработчиков.

Разработка ЗРС предполагает выполнение последовательности этапов, поочередное выполнение которых предполагает управление всеми процессами, происходящими в них.

\section{Управление}

Под управлением понимается процесс целенаправленного воздействия на объект управления со стороны управляющего объекта для изменения его состояния в соответствии с изменяющейся обстановкой [3].

Управление процессом создания предполагает решение ряда задач: синтеза, декомпозиции. Основной задачей системы управления является процесс формирования управляющих воздействий. Процесс управления совокупность действий, ведущая к образованию и совершенствованию связей между частями системы.

В процессе разработки ЗРС применяется ряд следующих требований к системе управления:

- информационная обеспеченность процесса управления;

- допустимые продолжительность цикла управления и уровень помех в нем;

- способность системы к выполнению своих функций во всем диапазоне внешних условий (общая живучесть системы);

- допустимая стоимость системы, включая расходы на ее создание и функционирование;

- специальные требования в зависимости от назначения системы.

Увеличение сложности задач управления боевыми действиями в современных условиях, конструктивной сложности выпускаемых изделий и повышение технологического уровня производства значительно усложняет решение задач планирования и оперативного управления процессами. 


\section{Разработка}

Поиск решений и обоснование разработки изделия осуществляется при выполнении проработок заказчика, прикладных научно-исследовательских работ (НИР), а доведение их результатов до условий промышленного освоения реализуется посредством выполнения опытно-конструкторской работы (ОКР).

Основным видом работ на стадии разработки является ОКР создания изделия. ОКР включает выполнение в необходимом объеме проектной и рабочей конструкторской документации, опытную проверку принятых технических решений и отработку этих решений в соответствии с потребностями производства и эксплуатации образцов техники.

Разработка сложных систем состоит в том, чтобы в заданный срок получить проект системы, обладающей максимальной ожидаемой эффективностью применения по назначению в условиях ограничения ресурсов на ее создание и эксплуатацию.

Основанием для проведения ОКР является тактико-техническое задание (ТТЗ), выданное заказчиком. ТТЗ на ОКР определяет цель разработки и назначение разрабатываемого (модернизируемого) образца, совокупность тактико-технических, технических, технико-экономических, специальных и других требований, этапы ОКР и, при необходимости, сроки их выполнения. На основании ТТЗ на ОКР разрабатываются технические задания на составные части ОКР.

ОКР проводится также, как и разработка ЗРС и состоит из следующих этапов:

— разработка эскизного проекта (ЭП);

- разработка технического проекта (ТП);

— разработка рабочей конструкторской документации (РКД) для изготовления опытного образца (OO);

- изготовление опытного образца и проведение предварительных испытаний (ПИ); 
— проведение государственных испытаний (ГИ) опытного образца (опытной партии);

— корректировка рабочей конструкторской документации и доработка опытного образца по результатам государственных испытаний.

Качество и степень обоснованности принятых решений на этапах ЭП и ТП определяют эффективность применения, стоимость и эксплуатационные свойства будущего изделия, продолжительность полезной части его жизненного цикла.

Высокое качество выполнения всех указанных мероприятий может быть достигнуто лишь при наличии специально организованного непрерывного научно-технического сопровождения разработки системы в течение всего его жизненного цикла.

Под научно-техническим сопровождением изделия понимают процесс планирования и выполнения заказчиком в тесном взаимодействии с предприятием разработчика научных, технических и организационных мероприятий, направленных на эффективное и своевременное решение задач разработки, испытаний и других задач, требующих целенаправленных действий по управлению качеством изделия на всех стадиях его жизненного цикла [4].

В процессе создания и использования ЗРС проходит определенную последовательность стадий жизненного цикла по каскадной модели.

Каскадная модель предусматривает последовательную организацию работ. При этом основной особенностью является разбиение всей разработки на этапы, причем переход с одного этапа на следующий происходит только после того, как будут полностью завершены все работы на предыдущем этапе. Каждый этап завершается выпуском полного комплекта документации, достаточной для того, чтобы разработка могла быть продолжена другой командой разработчиков [5].

За десятилетия существования модели «водопад» разбиение работ на стадии и названия этих стадий менялись. Кроме того, наиболее разумные методики и стандарты избегали жесткого и однозначного приписывания 
определенных работ к конкретным этапам. Тем не менее, все же можно выделить ряд устойчивых этапов разработки, практически не зависящих от предметной области (рис. 1):

- анализ требований заказчика;

- проектирование;

- разработка;

- тестирование и опытная эксплуатация;

- сдача готового продукта.

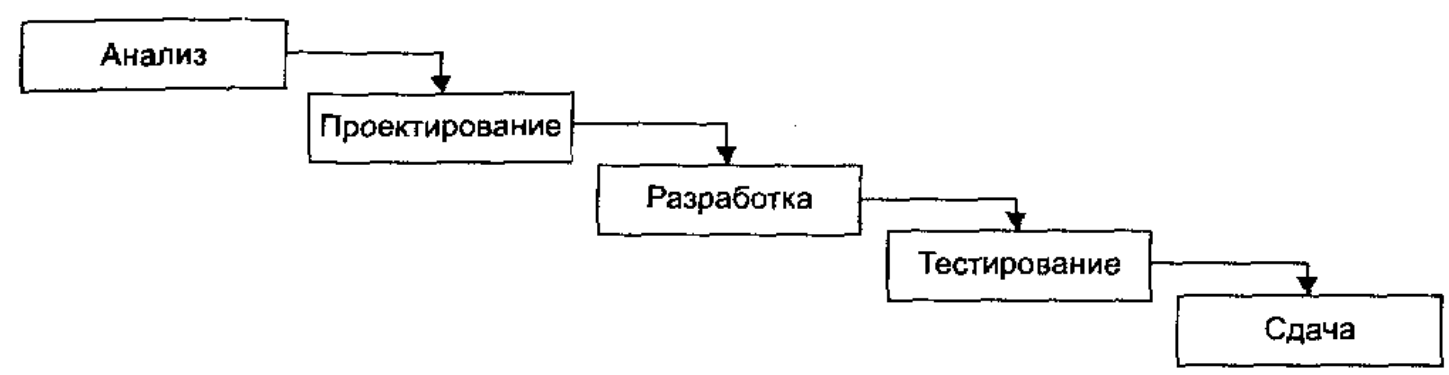

Рис. 1. Каскадная модель разработки

Достоинством классического жизненного цикла является то, что:

- на каждом этапе формируется законченный набор проектной документации, отвечающей критериям полноты и согласованности. На заключительных этапах также разрабатывается пользовательская документация, охватывающая все предусмотренные стандартами виды обеспечения системы (организационное, методическое, информационное, программное, аппаратное);

- выполняемые в логической последовательности этапы работ позволяют планировать сроки завершения и соответствующие затраты в соответствии с временным графиком по всем этапам проекта, упорядочивают ход конструирования.

Тем не менее, несмотря на все свои достоинства, каскадная модель имеет ряд недостатков: 
- реальные проекты часто требуют отклонения от стандартной последовательности шагов;

- цикл основан на точной формулировке исходных требований к изделию (реально в начале проекта требования заказчика определены лишь частично);

- результаты проекта доступны заказчику только в конце работы, что приводит к существенной задержке в получении результатов;

- ошибки и недоработки на любом из этапов проявляются, как правило, на последующих этапах работ, что приводит к необходимости возврата назад;

• сложность параллельного ведения работ по проекту;

- чрезмерная информационная перенасыщенность каждого из этапов;

- сложность управления проектом;

- высокий уровень риска и ненадежность инвестиций.

Проектно-исследовательские работы начинаются с установления необходимости создания новой системы и заканчиваются разработкой проектов элементов системы и испытанием опытных образцов этих элементов.

Спиральная модель, в отличие от каскадной, предполагает итерационный процесс разработки системы. При этом возрастает значение начальных этапов жизненного цикла, таких как анализ и проектирование. На этих этапах проверяется и обосновывается реализуемость технических решений путем создания прототипов.

Каждая итерация представляет собой законченный цикл разработки, приводящий к выпуску внутренней или внешней версии изделия (или подмножества конечного продукта), которое совершенствуется от итерации к итерации, чтобы стать законченной системой (рис. 2).

Таким образом, каждый виток спирали соответствует созданию фрагмента или версии программного изделия, на нем уточняются цели и характеристики проекта, определяется его качество, планируются работы следующего витка спирали. На каждой итерации углубляются и 
последовательно конкретизируются детали проекта, в результате чего выбирается обоснованный вариант, который доводится до окончательной реализации.

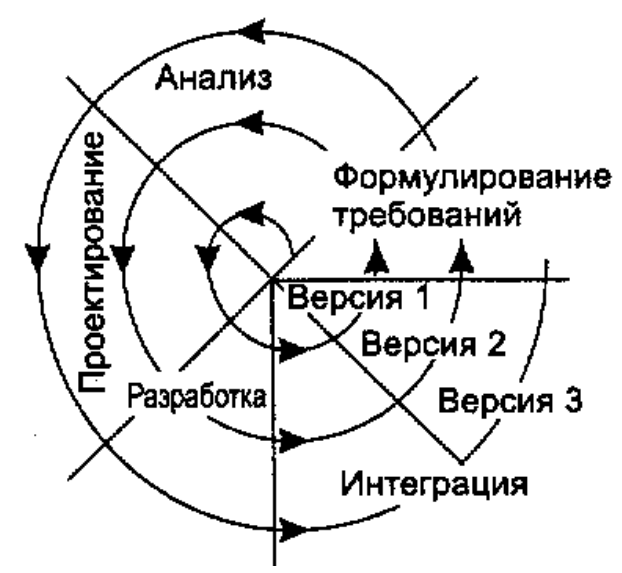

Рис. 2. Спиральная модель разработки

Использование спиральной модели позволяет осуществлять переход на следующий этап выполнения проекта, не дожидаясь полного завершения работы на текущем - недоделанную работу можно будет выполнить на следующей итерации. Главная задача каждой итерации — как можно быстрее создать работоспособный продукт, который можно показать пользователям системы. Таким образом, существенно упрощается процесс внесения уточнений и дополнений в проект.

Спиральный подход к разработке программного обеспечения позволяет преодолеть большинство недостатков каскадной модели и, кроме того, обеспечивает ряд дополнительных возможностей, делая процесс разработки более гибким.

Рассмотрим преимущества итерационного подхода более подробно:

- итерационная разработка существенно упрощает внесение изменений в проект при изменении требований заказчика;

- при использовании спиральной модели отдельные элементы зенитной ракетной системы интегрируются в единое целое постепенно. При итерационном подходе интеграция производится фактически непрерывно. 
Поскольку интеграция начинается с меньшего количества элементов, то возникает гораздо меньше проблем при ее проведении (по некоторым оценкам, при использовании каскадной модели разработки интеграция занимает до $40 \%$ всех затрат в конце проекта);

- уменьшение уровня рисков. Данное преимущество является следствием предыдущего, так как риски обнаруживаются именно во время интеграции. Поэтому уровень рисков максимален в начале разработки проекта. По мере продвижения разработки ожидаемый риск уменьшается. Данное утверждение справедливо при любой модели разработки, однако при использовании спиральной модели уменьшение уровня рисков происходит с наибольшей скоростью. Это связано с тем, что при итерационном подходе интеграция выполняется уже на первой итерации. И при выполнении начальных итераций выявляются многие аспекты проекта, такие как пригодность используемых инструментальных средств и программного обеспечения, квалификация разработчиков и т.п. На рис. 3 приведены в сравнении графики зависимости уровня рисков от времени разработки при использовании каскадного и итерационного подходов;

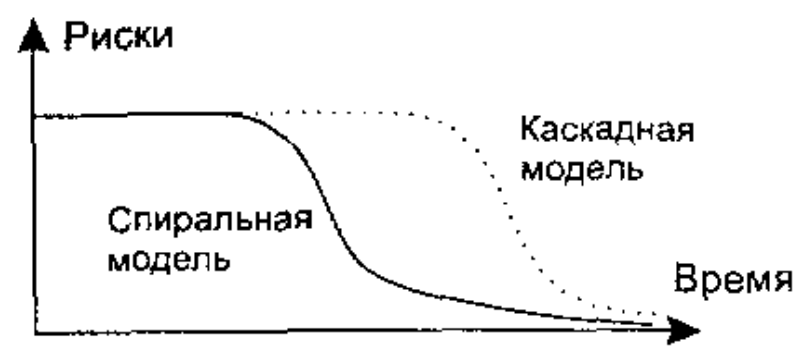

\section{Рис. 3. Зависимость рисков от времени разработки}

- итерационная разработка обеспечивает большую гибкость в управлении проектом, давая возможность внесения тактических изменений в разрабатываемое изделие. Например, можно сократить сроки разработки за счет уменьшения функциональности системы или использовать в качестве составных частей системы продукцию сторонних фирм вместо собственных 
разработок. Это может быть актуальным в условиях конкурентной борьбы, когда необходимо противостоять продвижению изделия, предлагаемого конкурентами;

- итерационный подход упрощает повторное использование компонентов и позволяет использовать компонентный подход к программированию. Это обусловлено тем, что гораздо проще выявить (идентифицировать) общие части проекта, когда они уже частично разработаны, чем пытаться выделить их в самом начале проекта. Анализ проекта после проведения нескольких начальных итераций позволяет выявить общие, многократно используемые компоненты, которые на последующих итерациях будут совершенствоваться;

- спиральная модель позволяет получить более надежную и устойчивую систему. Это связано с тем, что по мере развития системы ошибки и слабые места обнаруживаются и исправляются на каждой итерации. Одновременно могут корректироваться критические параметры эффективности, что при использовании каскадной модели выполняется только перед внедрением системы;

- итерационный подход позволяет совершенствовать процесс разработки;

- анализ, проводимый в конце каждой итерации, позволяет проводить оценку того, что должно быть изменено в организации разработки, и улучшить ее на следующей итерации.

Основная проблема спирального цикла - определение момента перехода на следующий этап. Для ее решения необходимо ввести временные ограничения на каждый из этапов жизненного цикла. Иначе процесс разработки может превратиться в бесконечное совершенствование уже сделанного. При итерационном подходе полезно следовать принципу «лучшее - враг хорошего». Поэтому завершение итерации должно производиться строго в соответствии с планом, даже если не вся запланированная работа закончена.

Планирование работ обычно проводится на основе статистических данных, полученных в предыдущих проектах, и личного опыта разработчиков. 


\section{Планирование}

Этап планирования разработки системы выполняется заказчиком системы. Он включает тактико-технико-экономическое обоснование системы и разработку наиболее общих требований к системе. Этап начинается с установления необходимости и целесообразности создания новой системы, формирования ее назначения и решаемых задач. На этом этапе определяются основные ограничения на технические, организационные и экономические факторы, при которых в дальнейшем будет разрабатываться система. Результаты исследований, полученные на первом этапе, позволяют приступить к проектированию системы. Работы на этом этапе проводятся разработчиком в условиях, когда к проектируемой системе уже предъявлены отдельные требования и определены ограничения.

\section{Проектирование}

Проектирование предполагает выполнение двух основных направлений:

- выбор технических решений;

- отображение этих решений в виде совокупности документов для изготовления объекта в процессе производства.

Процесс проектирования ЗРС, как процесс принятия решения, принято делить на внешнее проектирование и проектирование внутреннее. Для внешнего проектирования основной проблемой является формулирование целей, для достижения которых создается система, и задач, которые она должна решать в процессе функционирования, чтобы обеспечить достижение поставленных целей, а также предъявление требований к основным характеристикам системы, выполнение которых обеспечивает решение задач.

Основа внешнего проектирования - правильный учет современного состояния техники, возможностей технологии, обоснованный прогноз их развития на период времени, не меньший планируемой продолжительности жизненного цикла системы. Наряду с техническими факторами необходимы учет экономических факторов, прогноз стоимости системы и сроков её разработки и изготовления. На основе изучения состояния и перспектив 
научно-технического прогресса формулируется первоначальный вариант тактико-технического задания на систему.

Проблема внутреннего проектирования состоит в том, чтобы реализовать в виде комплекса технических устройств, узлов и агрегатов, составляющих систему, основные технические параметры, которые обеспечат системе требуемые свойства и характеристики. Часто требования, вытекающие из внешнего проектирования, противоречат возможностям внутреннего проектирования. Выражается это в том, что либо они завышены и поэтому не выполнимы, либо занижены и не используют всех достижений научнотехнического прогресса. Возникает проблема формирования такого облика системы, при котором требования внешнего проектирования оказались бы корректно согласованными с возможностями внутреннего проектирования.

В процессе взаимодействия внешнего и внутреннего проектирования первоначально рождается облик системы, который затем воплощается в проектные решения.

При внешнем и внутреннем проектировании происходит постановка и решение основной задачи проектирования - задачи синтеза технической системы, удовлетворяющей заданным требованиям.

Процесс проектирования реализуется в соответствии с определенным планом, который представляется в виде логической схемы построения проекта. Общая технология проектирования сложных систем предусматривает предварительное, эскизное и техническое проектирование.

На этапе эскизного проектирования производится уточнение и конкретизация структурной схемы системы, а также детальный анализ характеристик используемых технических средств. С этой целью решаются задачи инженерного синтеза и анализа системы. Основной задачей эскизного проектирования является уточнение параметров и характеристик системы, связанное с проектно-конструкторской проработкой её основных подсистем и формированием их облика, осуществляемое на основе иерархической уровневой структуризации системы. Данный этап сопровождается проведением 
широкого комплекса расчетов, моделирования и экспериментальных исследований. Результатом этапа является эскизный проект. Объект на этапе ЭП рассматривается как единое целое на абстрактном алгоритмическом уровне. В задачи этапа входят два класса задач: внутренние и внешние. К внутренним относится отработка структуры объекта, системных алгоритмов, протоколов обмена информацией и интерфейса. К внешним относятся задачи управления последующей обработкой, связанные с выработкой совокупности частных требований к элементам структуры, к определениям внешних условий функционирования элементов.

Технический проект завершает проектирование образца и представляет собой совокупность конструкторских документов, которые содержат технические решения, дающие полное представление об устройстве изделия и исходные данные для разработки рабочей конструкторской документации. При создании сложных технических комплексов предлагается корректировать технический проект с учетом старения идеи, положенной в его основу, уже в процессе проектирования и создания системы; рекомендуют при создании технической документации, сопровождающей систему, включать в нее не только этапы разработки и эксплуатации системы, но и срок жизни, ликвидацию.

На этапе технического проектирования глубина проработки проекта достигает уровня базовых элементов. Результатом этого этапа является технический проект.

В результате выполнения этого этапа синтезируются структуры, алгоритмы управления, а также формируются требования к элементам структуры и условия их функционирования.

Рабочая конструкторская документация необходима для изготовления и проведения испытаний опытного образца. Она корректируется по результатам изготовления этого образца и его предварительных и государственных испытаний. Целью корректировки является подготовка документации для серийного производства изделия. 
Существующее в единой системе конструкторской документации (ЕСКД) деление схем на принципиальные, функциональные и структурные отражает блочно-иерархический подход к проектированию. Принципиальные схемы определяют полный набор базовых элементов и связей между ними и обычно дают детальное представление о принципах работы изделия.

Функциональные схемы разъясняют протекание процессов в изделии или его частях, т.е. дают представление о функционировании объекта с учетом только существенных факторов и функциональных частей.

Структурные схемы отражают наиболее общее и наименее детализированное представление об объекте, определяя основные функциональные части изделия, их назначение и взаимосвязи.

Этап конструкторского проектирования обеспечивает конструкторское оформление всех решений, принимаемых на предыдущих этапах. Центральной задачей этапа является синтез геометрической модели объекта проектирования на основе проектных процедур. В состав проектных процедур входят операции компоновки, размещения, определения геометрических форм объектов, синтеза оригинальных деталей. Геометрическая модель отображает объект в элементах конструкторского базиса в форме, удобной и достаточной для формирования полной конструкторской документации.

В процессе проектирования системы используется декомпозиция. Декомпозиция проектных работ может быть этапной, т.е. такой, когда один и тот же объект проходит ряд этапов, на каждом из которых решаются те или иные проектные задачи. Внутри этапа также возможна декомпозиция, которая предполагает расчленение самого объекта на ряд самостоятельно проектируемых объектов. В инженерной практике декомпозиция по этапам связана с классификацией проектных работ. Внутренняя декомпозиция связана с классами аппаратуры или иерархии объектов.

Эвристическая деятельность опирается на имеющийся опыт в разработке подобных систем или решении подобных задач и на результаты теоретических и экспериментальных исследований, проводимых в процессе проектирования. 
Экспериментальные методы проектирования, прежде всего, основаны на методах макетирования. На макете - физической модели - определяется выполнение условий работоспособности, производится изменение внутренних параметров, частичное изменение структуры с целью улучшения свойств объекта.

На упрощение и ускорение проектирования ориентирован принцип типизации и унификации проектных решений и средств проектирования. Унификация целесообразна только в таких классах объектов, в которых из сравнительно небольшого числа разновидностей элементов предстоит проектирование и изготовление большого числа разнообразных систем. Именно эти разновидности элементов и подлежат унификации. Для сложных систем, состоящих из этих элементов, в каждом конкретном случае приходится заново выполнять многоуровневое иерархическое проектирование. В таких условиях целесообразно ставить вопрос не только об унификации изделий, а также и об унификации средств проектирования и изготовления, в частности, об унификации проектных процедур в рамках автоматизированного выполнения типовых проектных процедур, что позволяет более оперативно создавать проекты новых изделий.

Проектирование завершается изготовлением опытных образцов объекта проектирования. Изготовленные по рабочей конструкторской документации опытные образцы подвергаются предварительным и приемочным (государственным) испытаниям. Эти испытания проводят для проверки соответствия образцов требованиям, указанным в ТТЗ, действующей нормативно-технической документации и для обоснования предложений о возможности постановки разрабатываемых изделий на производство и принятия в эксплуатацию.

\section{Испытания}

Предварительные испытания выполняются под руководством головного разработчика изделия. Они относятся к контрольным испытаниям и проводятся с целью доводки опытного образца до полного удовлетворения предъявленным 
требованиям, предварительного оценивания боевых, технических и эксплуатационных характеристик образца и установления их соответствия требованиям ТТ3, а также для определения готовности образца к приемочным испытаниям. Основной задачей доводочных испытаний является:

- выявление и устранение в опытных образцах изделий конструктивных и производственных дефектов;

- взаимная настройка всех систем, входящих в изделия, и доведение их до полного соответствия предъявленным требованиям;

- окончательная отработка конструкции изделия и технологии его производства;

- оценивание контролепригодности изделия в производстве и эксплуатации, а также отработка состава штатных средств контроля и испытаний.

Приемочные испытания образцов техники и их составных частей, разрабатываемых по ТТЗ заказчика, являются государственными испытаниями, которые организует заказчик, несущий ответственность за их проведение. Их выполняют во всем диапазоне условий и режимов эксплуатации, указанных в TТ3 и конкретизированных в программе и методиках испытаний. Условия проведения испытаний максимально приближают к условиям реальной эксплуатации и боевого применения изделий. Целью государственных испытаний является проверка и подтверждение соответствия боевых, технических и эксплуатационных характеристик образца требованиям ТТЗ, а также выработка рекомендаций по боевому применению изделия и возможности принятия его на вооружение и постановки на производство.

Государственные испытания опытного образца и эксплуатационные испытания головных образцов опытной серии изделия по существу являются завершающими испытаниями изделия опытного производства, после которых оно переходит на следующую стадию своего жизненного цикла - стадию серийного производства, для которой характерны различные категории испытаний серийной продукции. 


\section{Эксплуатация}

На стадиях эксплуатации и капитального ремонта для устранения неисправностей и восстановления технического ресурса изделия подвергаются текущему, среднему и капитальному ремонту. После ремонта проводится комплексный контроль, регулировка и испытания средствами и методами, аналогичными производственным испытаниям. В процессе ремонтных испытаний выявляются неисправности, которые не были обнаружены в процессе контрольных операций при ремонте и сборке изделий. Далее осуществляется цикл регулировочных операций для обеспечения требуемых значений характеристик изделий. После этого проводится окончательный контроль изделий и передача их в эксплуатацию или перевод в режим нормального функционирования в соответствии с назначением изделий.

\section{Заключение}

Таким образом, для создания сложной системы необходимо решить задачу синтеза системы управления (разработки, совершенствования или реорганизации структуры системы), которая должна обладать желаемыми свойствами.

Типовыми требованиями, предъявляемыми к системе управления, являются:

- информационная обеспеченность процесса управления;

- допустимые продолжительность цикла управления и уровень помех в нем;

- защищенность контура управления от внешних помех;

- ограничения допустимой величиной вероятность нарушения цикла управления во всем диапазоне условий функционирования системы, что означает фиксацию допустимой вероятности отказов при выполнении целевых функций системы;

- способность системы к выполнению своих функций во всем диапазоне внешних условий, т.е. общая живучесть системы; 
- структура системы управления должна обеспечить запас статической устойчивости к малым возмущениям внешней и внутренней сред, демпфируя их в местах воздействия на систему и препятствуя развитию эффектов отрицательной синергии при распространении их воздействия на другие подсистемы;

- допустимая стоимость системы, включая расходы на её создание и функционирование;

- специальные требования в зависимости от назначения системы.

Иногда в эти требования включают число управляемых объектов, которые должна вести подсистема управления, однако для системы это требование не является определяющим, поскольку укрупнением либо разукрупнением управляемых объектов можно изменить их число в нужном направлении. Наоборот, определение числа и состава входящих в систему подсистем (компонентов), а также способов организации связей между ними и составляет типичную задачу реорганизации системы при её переходе на следующий этап развития [1].

\section{Список литературы}

1. Ашурбейли И.Р. Сложные радиоэлектронные системы вооружения. Планирование и управление созданием / И.Р. Ашурбейли, А.И. Лаговиер, С.П. Соколов // Радиотехника. Москва, 2010. - С. 50-52.

2. Баштанник Н.А. Теоретические и методологические аспекты развития современной науки / Н.А. Баштанник, А.Н. Баштанник, Т.Н. Светличкина // Коллективная монография. Москва, 2020. - Том 8. - С. 87-103.

3. Баштанник Н.А. Метод оценки телекоммуникационной сети обмена данными перспективных АСУ с использованием технико-экономических показателей / Н.А. Баштанник, В.И. Лобейко // Материалы Международной научной конференции 11-14 мая 2010. Инновационные технологии в управлении, образовании, промышленности «АСТИНТЕХ-2010». Астрахань. - 2010. - C. 6-8. 
4. Баштанник Н.А. Оценка пропускной способности телекоммуникационной сети обмена данными АСУ методом Монте-Карло / Н.А. Баштанник, В.И. Лобейко, С.В. Поляков // Материалы Международной научно-практической конференции. Инновации на основе информационных и коммуникационных технологий «ИНФО - 2010». Россия, Сочи. 1 - 10 октября. 2010. - C. 320-323.

5. Лобейко В.И. Современные подходы к организации испытаний сложных систем. - Астрахань: Астраханский ун-т, 2006. - 367 с.

(C) Н.А. Баштанник, В.И. Лобейко, Н.Г. Мустафаев, И.С. Погребняк, 2021 